\title{
Sphere-forming cell subpopulations with cancer stem cell properties in human hepatoma cell lines
}

\author{
Lu Cao ${ }^{1}$, Yanming Zhou ${ }^{2}$, Beibei Zhai ${ }^{1}$, Jian Liao ${ }^{1}$, Wen Xu' ${ }^{1}$, Ruixiu Zhang ${ }^{1}$, Jing Li ${ }^{1}$, Yu Zhang ${ }^{1}$, Lei Chen ${ }^{1}$, \\ Haihua Qian', Mengchao $\mathrm{Wu}^{1}$ and Zhengfeng Yin ${ }^{1 *}$
}

\begin{abstract}
Background: Cancer stem cells (CSCS) are regarded as the cause of tumor formation and recurrence. The isolation and identification of CSCs could help to develop novel therapeutic strategies specifically targeting CSCs.

Methods: Human hepatoma cell lines were plated in stem cell conditioned culture system allowed for sphere forming. To evaluate the stemness characteristics of spheres, the self-renewal, proliferation, chemoresistance, tumorigenicity of the PLC/PRF/5 sphere-forming cells, and the expression levels of stem cell related proteins in the $\mathrm{PLC/PRF} / 5$ sphere-forming cells were assessed, comparing with the parental cells. The stem cell RT-PCR array was performed to further explore the biological properties of liver CSCs.

Results: The PLC/PRF/5, MHCC97H and HepG2 cells could form clonal nonadherent 3-D spheres and be serially passaged. The PLC/PRF/5 sphere-forming cells possessed a key criteria that define CSCs: persistent self-renewal, extensive proliferation, drug resistance, overexpression of liver CSCs related proteins (Oct3/4, OV6, EpCAM, CD133 and CD44). Even 500 sphere-forming cells were able to form tumors in NOD/SCID mice, and the tumor initiating capability was not decreased when spheres were passaged. Besides, downstream proteins DTX1 and Ep300 of the CSL (CBF1 in humans, Suppressor of hairless in Drosophila and LAG1 in C. elegans) -independent Notch signaling pathway were highly expressed in the spheres, and a gamma-secretase inhibitor MRK003 could significantly inhibit the sphere formation ability.
\end{abstract}

Conclusions: Nonadherent tumor spheres from hepatoma cell lines cultured in stem cell conditioned medium possess liver CSC properties, and the CSL-independent Notch signaling pathway may play a role in liver CSCs.

\section{Background}

Hepatocellular carcinoma (HCC) is the fifth most common cancer and the third leading cause of cancer death worldwide [1,2]. Its overall incidence remains alarmingly high in the developing countries and is steadily rising across most of the developed countries [1-3]. Although the cytologic pathogenesis of HCC remains unclear, it has been proposed that only a small fraction of cancer cells with stem cell properties, named cancer stem cells (CSCs), is responsible for the initiation, progression, local and distant recurrence/metastasis of HCC, also for the failure of chemo- and radiotherapy. The clinical corollary of this hypothesis has been extended to proposals to treat cancer by targeting the putative liver CSCs.

\footnotetext{
*Correspondence: yinzfk@yahoo.com.cn

'Department of Molecular Oncology, Eastern Hepatobiliary Surgery Hospital, Second Military Medical University, Shanghai, China

Full list of author information is available at the end of the article
}

Numerous attempts have been made to identify cells with stem cell properties in established HCC cell lines. Different research groups have reported that liver CSC fractions could be successfully enriched by some cell surface phenotypes, specifically CD133, CD90, CD44, epithelial cell adhesion molecule (EpCAM), OV6 and CD13 [4-9]. Nevertheless, these proteins, which are involved in embryonic and somatic stem cell function, embryonic development, hepatocyte membrane transport and growth control, have been demonstrated a relative lack of sensitivity and specificity for identifying liver CSCs [10-12]. So far, no markers for putative liver CSCs have yet been generally accepted, and further study is needed to explore the isolation method for liver CSCs.

A major advance in adult stem cell research was achieved in 1996 when it was discovered that the undifferentiated multipotent neural cells could be grown and maintained in suspension using the neurosphere assay
C Biomed Central

() 2011 Cao et al; licensee BioMed Central Ltd. This is an Open Access article distributed under the terms of the Creative Commons Attribution License (http://creativecommons.org/licenses/by/2.0), which permits unrestricted use, distribution, and reproduction in any medium, provided the original work is properly cited. 
[13]. Then anchorage-independent sphere culture of stem cells was instrumental in the study of adult stem cells including the nerve, prostate and mammary stem cells [14-17]. Recently, as a functional approach, sphere formation is particularly useful to enrich the potential CSC subpopulations when the specific CSC makers have not been defined as the case for most CSCs [18-25]. However, there have been few reports for sphere culture in liver cancer. Therefore, the present study intends to establish an alternate approach to isolate, identify and characterize liver cancer cell subsets with CSC properties.

\section{Methods}

\section{Cell lines and sphere culture}

Human hepatoma cell lines, PLC/PRF/5 and HepG2, were obtained from the Cell Bank of Chinese Academy of Sciences (Shanghai, China). MHCC97H was obtained from the Liver Cancer Institute, Zhongshan Hospital, Fudan University (Shanghai, China). All of the cells were maintained as a monolayer in high glucose DMEM with $10 \%$ fetal bovine serum (FBS), $100 \mathrm{IU} / \mathrm{ml}$ penicillin $\mathrm{G}$ and $100 \mu \mathrm{g} / \mathrm{ml}$ streptomycin at $37^{\circ} \mathrm{C}$ in a humidified $5 \% \mathrm{CO}_{2}$ incubator. Cells were collected and washed to remove serum, then suspended in serum-free DMEM/F12 supplemented with $100 \mathrm{IU} / \mathrm{ml}$ penicillin, $100 \mu \mathrm{g} / \mathrm{ml}$ streptomycin, $20 \mathrm{ng} / \mathrm{ml}$ human recombinant epidermal growth factor (hrEGF), $10 \mathrm{ng} / \mathrm{ml}$ human recombinant basic fibroblast growth factor (hrbFGF), 2\% B27 supplement without vitamin A, 1\% N2 supplement (Invitrogen, Carlsbad, CA, USA). The cells were subsequently cultured in ultra low attachment 6-well plates (Corning Inc., Corning, NY, USA) at a density of no more than 5,000 cells/well.

\section{Sphere passage and sphere formation assay}

The spheres were collected by gentle centrifugation, then dissociated with trypsin-EDTA and mechanically disrupted with a pipette. The resulting single cells were then centrifuged to remove the enzyme and re-suspended in serum-free medium allowed to re-form spheres. The spheres should be passaged every 5-8 days before they reached a diameter of $100 \mu \mathrm{m}$. The dissociated single sphere-forming cells were also diluted to a density of $500 \mathrm{cells} / \mathrm{ml}$. Then, the $2 \mu \mathrm{l} /$ well diluted cell suspension was plated to ultra low attachment 96-well plate (Corning Inc., Corning, NY, USA), and $150 \mu \mathrm{l}$ of serum-free medium was added. The wells with only one cell were marked and observed everyday.

\section{Colony formation assay}

The PLC/PRF/5 spheres were enzymatically dissociated as described above. Trypan blue staining was used to determine cell viability, and more than $95 \%$ of cells with viability were acceptable for the following experiments.
The single cells were seeded in DMEM with 10\% FBS at a density of 2000 cells/well on 6-well plates that were pre-coated with Matrigel (BD Biosciences, San Jose, CA, USA). After 7 days, the colony formation ability was assessed by counting the number of colonies ( $>70$ cells) under a microscope after crystal violet staining (SigmaAldrich, St. Louis, MO, USA). Representative views were photographed. The parental cells were plated at the same density as the control.

\section{Chemotherapy sensitivity assays}

The sensitivity of the PLC/PRF/5 parental and sphereforming cells to chemotherapeutic drugs was measured by MTT assay. Briefly, cells were seeded in 96-well plates that were precoated with Matrigel, and various concentrations of cisplatin (Sigma-Aldrich) were added at the beginning, co-incubated for $12 \mathrm{~h}$ or $24 \mathrm{~h}$. After changing to fresh medium without cisplatin, cells were cultured for another $72 \mathrm{~h}$. The MTT reagent (SigmaAldrich) was then added to each well according to the manufacturer's instructions. Absorbance was measured at $490 \mathrm{~nm}$.

\section{Immunofluorescent staining}

Cells were fixed in $4 \%$ paraformaldehyde and blocked with normal goat serum. The primary antibodies, including mouse anti-human OV6 (R\&D Systems Inc., Minneapolis, MN, USA), mouse anti-human CD133 and rabbit anti-human CD44 (Santa Cruz Biotechnology, Inc. Santa Cruz, CA. USA) were added and incubated overnight at $4{ }^{\circ} \mathrm{C}$. After washing 3 times with PBS, the goat anti-mouse IgG and goat anti-rabbit secondary antibodies conjugated with Cy3, FITC or TRITC (Jackson ImmunoResearch Laboratories Inc., West Grove, PA, USA) were added and incubated at room temperature for $1 \mathrm{~h}$. Cells were then counterstained with DAPI (Sigma-Aldrich) and the images were captured using an Olympus-IX71 fluorescent microscope (Olympus Inc., Center Valley, PA, USA).

\section{In vivo tumorigenicity experiments}

All mice were cared for in accordance with institutional guidelines. The PLC/PRF/5 parental and the third, sixth and ninth passages of sphere-forming cells were used in tumorigenicity experiments. Trypan blue staining was used to assess cell viability, and various numbers of viable single cells were subcutaneously injected into 5week-old NOD/SCID male mice (Shanghai Laboratory Animal Center, Chinese Academy of Sciences, Shanghai, China) in serum-free DMEM/Matrigel (1:1) using $100 \mu \mathrm{l}$ microsyringe. Mice were killed at 8 weeks after cell injection, then the tumors were harvested for further examination. 


\section{Real-time PCR microarray analysis}

Human Stem Cell $\mathrm{RT}^{2}$ ProfilerTM PCR Array (PAHS405A, SABioscience, USA) was done according to the manufacturer's instructions. Briefly, RNA samples from both the PLC/PRF/5 parental cells (as control) and the tertiary passage spheres were prepared. After removal of contaminating DNA from RNA preparations, total RNA samples were cleaned up and the resulting RNA were assessed for both yield and quality. After the first strand cDNA was synthesized, real-time PCR was performed. The data were analyzed using the $\Delta \Delta \mathrm{Ct}$ method.

\section{Western blotting analysis}

Quantified protein lysates were resolved on SDS-PAGE gels, transferred onto a polyvinylidene difluoride membrane (Millipore, Billerica, MA, USA), and immunoblotted with the primary antibodies against EpCAM, CD133, activated Notch1, Ep300 (Abcam, Cambridge, MA, USA), DTX1, Oct3/4 (Santa Cruz, CA, USA), or OV6, CD44 (R\&D Systems Inc., MN, USA), followed by incubation with the horseradish peroxidase-conjugated secondary antibody. The blots were visualized using a supersignal west femto maximum sensitivity substrate kit (Pierce, Waltham, MA, USA). GAPDH was used as a loading control.

\section{Statistical analysis}

All values in the figures and text were showed as means \pm SD. Statistical analyses were performed using the SPSS statistical software package (SPSS/PC+, SPSS Inc., Chicago, IL, USA). Any significant differences among mean values were evaluated by the student's $t$ test. A two-sided $P<0.05$ was accepted as significant.

\section{Results}

Hepatoma cells could form anchorage-independent, selfrenewing spheres

The hepatoma cells were plated in stem cell conditioned culture medium in 6-well plates at a density of 5,000 cells/well which allowed for the formation of colonies separated from each other. In this condition, cells grew as nonadherent, three-dimensional sphere clusters, called spheres. Figure 1A showed anchorage-independent spheres formed by the HepG2, MHCC97H and PLC/ $\mathrm{PRF} / 5$ cells. After 5 to 8 days, when the spheres grew to 70 to $100 \mu \mathrm{m}$ in diameter, they were passaged and the

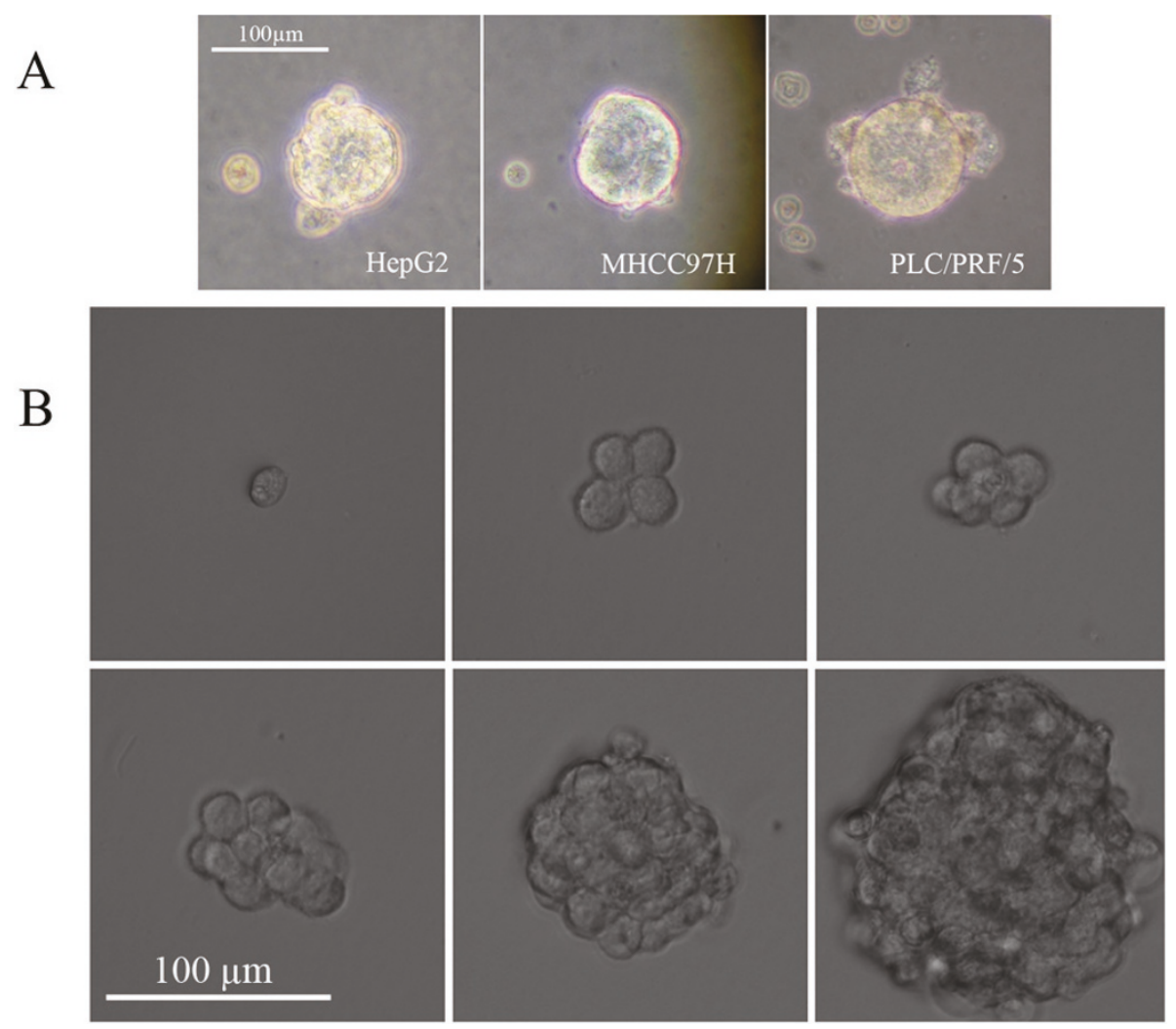

Figure 1 Hepatoma cells formed the anchorage-independent, self-renewing spheres. (A) Hepatoma cell lines, including HepG2, MHCC97H and PLC/PRF/5, could form the anchorage-independent 3-D spheres in stem cell conditioned culture medium (200x). (B) Generation of a sphere from a single PLC/PRF/5 cell. The propagation of a single cell cultured in a 96-well dish was recorded at day 1, 3, 5, 7, 9 and 13, separately $(400 x)$. 
single cell from spheres could propagate to form new spheres again. A key property of all normal and cancer stem cells is their unique ability to self-renew. One of the methods to determine the self-renewal capacity of sphere-forming cells is to test their capability of serial passage. The PLC/PRF/5, HepG2 and MHCC97H spheres had been serially passaged for more than 12 generations, indicating their self-renewal capability in vitro.

To corroborate the finding that a sphere could be generated from a single cell, one PLC/PRF/5 cell per well was plated to a 96-well plate and the wells with one cell were visualized everyday. Figure $1 \mathrm{~B}$ showed the process of single PLC/PRF/5 cell forming a sphere.

\section{Sphere-forming cells proliferate extensively in vitro}

We compared the proliferative ability of the tertiary passage PLC/PRF/5 spheres and its parental cell line using clonogenicity assay. Both of them were adherently plated and were alive, but were unable to form comparable colonies. The sphere-forming cells proliferated significantly faster and induced bigger and greater numbers of tumor colonies than the parental cells. Based on counting the number of colonies per 2,000 seeded cells, it was $175.67 \pm 30.07 / 381.00 \pm 61.02(P=0.006)$ for the parental/sphere-forming PLC/PRF/5 cells (Figure $2 \mathrm{~A}$ ). The sphere-forming cells were capable of extensive proliferation, indicating that the sphere-forming cells could play an important role in the maintenance of tumor growth.

\section{Sphere-forming cells possess the ability of resistance to conventional chemotherapy in vitro}

HCC cells are commonly resilient to chemotherapy. It is speculated that cancer stem or progenitor cells in HCC are more resistant to conventional chemotherapy contributing to recurrence. To examine whether the selfrenewing sphere-forming cells possess a hypothesized CSC chemoresistant property, the sensitivity of the PLC/PRF/5 parental cells versus the tertiary passage sphere-forming cells to cisplatin was assessed. The survival rates of sphere-forming cells were higher under the treatment of $3 \mu \mathrm{g} / \mathrm{ml}, 5 \mu \mathrm{g} / \mathrm{ml}$ and $9 \mu \mathrm{g} / \mathrm{ml}$ cisplatin for $12 \mathrm{~h}$, being 1.4-fold, 1.9-fold, 1.8-fold, respectively, compared with the parental PLC/PRF/5 cells $(P<0.01)$, whereas, under the treatment for $24 \mathrm{~h}$, the relative survival rates were increased to 1.5-fold, 2.1-fold and 2.3fold respectively (Figure $2 \mathrm{~B}, \mathrm{P}<0.01$ ).

We also tested the sensitivity of sphere-forming cells to other 4 drugs in addition to cisplatin. The PLC/PRF/ 5 sphere-forming cells exhibited general resistance to 5$\mathrm{Fu}$, gemcitabine, mitomycin and sorafenib in the treatment of 36 h. Compared with the PLC/PRF/5 parental cells, the survival rates of PLC/PRF/5 sphere-forming cells were higher under $200 \mu \mathrm{mol} / \mathrm{L}, 400 \mu \mathrm{mol} / \mathrm{L}$ of 5 Fu (1.60-fold, 1.98-fold respectively, $P<0.01) ; 5 \mathrm{mmol} /$
$\mathrm{L}, 10 \mathrm{mmol} / \mathrm{L}$ of gemcitabine (1.99-fold, 2.49-fold respectively, $P<0.01) ; 0.5 \mu \mathrm{mol} / \mathrm{L}, 1.0 \mu \mathrm{mol} / \mathrm{L}$ of mitomycin (1.24-fold, 2.33-fold respectively, $P<0.01$ ); and 6 $\mu \mathrm{mol} / \mathrm{L}, 12 \mu \mathrm{mol} / \mathrm{L}$ of serafenib (2.07-fold, 15.21-fold respectively, $P<0.01$ ). The results support a role for these sphere-forming cells in HCC chemoresistance, which may explain why current therapies fail to eradicate progenitors and prevent tumor re-growth.

\section{Sphere-forming cells exhibit high tumorigenicity in vivo}

To confirm that the sphere-forming cells exhibit greater tumor initiating capability, NOD/SCID mice were transplanted with varying amounts of the PLC/PRF/5 sphereforming cells ranging from the amount that is unable to initiate tumor growth to the amount that always initiates tumor formation. The PLC/PRF/5 parental cells were operated as controls. As few as 500 sphere-forming cells were sufficient for tumor development, whereas, at least $2 \times 10^{5}$ parental cells were necessary to consistently generate a tumor in the same model, and not to mention, requiring a longer period of time (Table 1 Figure $3)$. The tumor nodules formed by the PLC/PRF/5 sphere-forming cells displayed similar histology to that by the parental cells. To inspect whether the tumor initiating capability could be decreased as the spheres were passaged, we also compared the tumorigenicity of different generations of spheres. The results showed that both the tumor initiating capability and phenotypic appearance were similar for the 3th, 6th and 9th generations of sphere-forming cells (Table 1). The tumorigenic efficacies of three cell lines HepG2, PLC/PRF/5 and $\mathrm{MHCC} 97 \mathrm{H}$ were also compared in nude mice (Additional file 1 Figure S1). The results suggested that the tumorigenic efficacies of sphere-forming cancer cells were enhanced compared with the parental cells, and the volumes of tumors were positively correlated with malignant grade of the cell lines (malignant grade HepG2 < PLC/PRF/5 < MHCC97H). Interestingly, the HepG2 parental cells at $10^{6}$ cells/mouse could not form visible xenografts $(0 / 5)$, but the HepG2 sphere-forming cells at $10^{6}$ cells/mouse could form xenograft tumors in the same period of 30 days.

\section{Sphere-forming cells overexpress liver CSC related proteins and the CSL-independent Notch signaling pathway might play a role in liver CSCs}

To date, anti-OV6, a monoclonal antibody raised against cells isolated from carcinogen treated rat liver [26], remains the best available marker of hepatic stem cells [27], even though it also reacts with bile duct epithelium in rats and humans [28]. Besides, Yang et al reported that the $\mathrm{OV}^{+}$liver cancer cells may represent a potential stem/progenitor-like cell population [8]. Immunofluorescent staining for OV6 showed that most of the PLC/PRF/ 


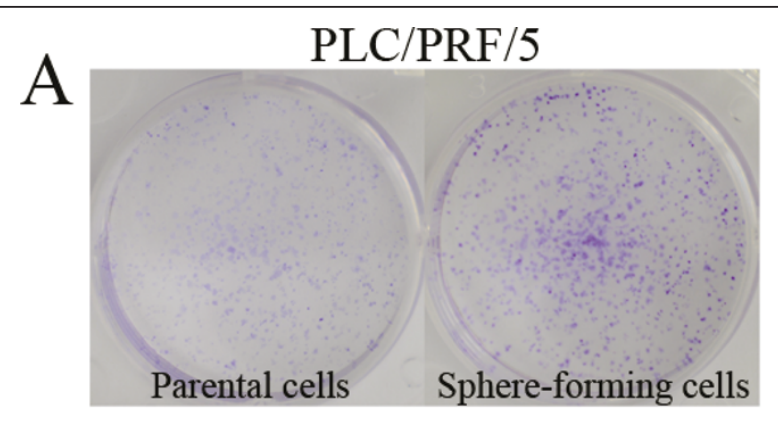

$\mathrm{B}$
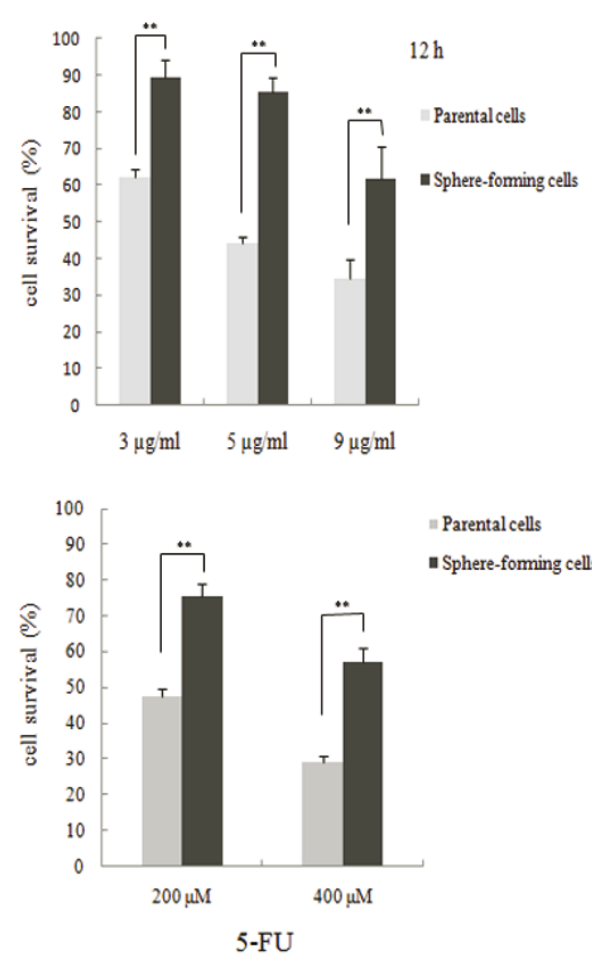

Parental cells

Sphere-forming cells

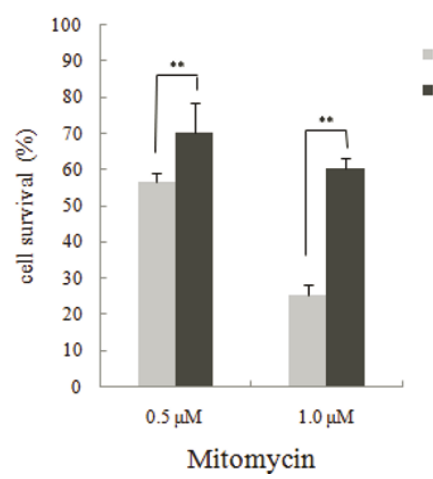

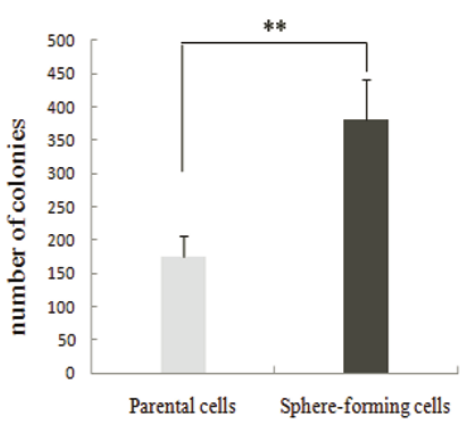
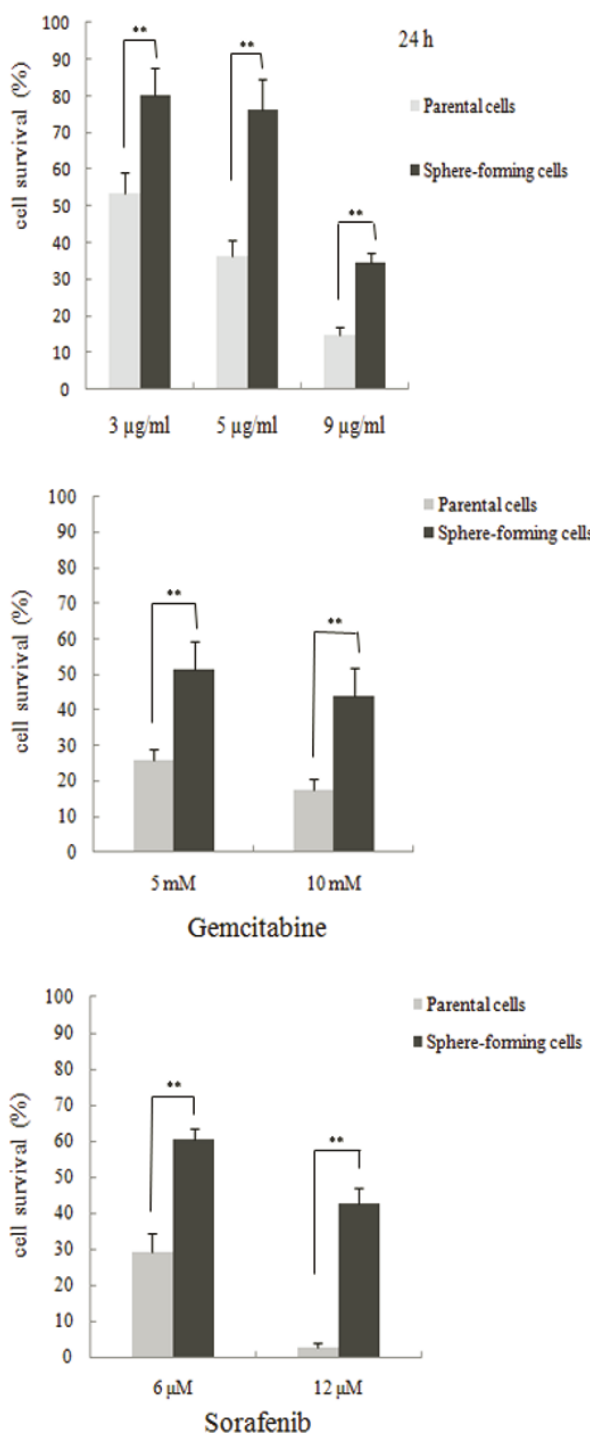

Figure 2 Sphere-forming cells proliferated extensively and possessed resistance ability to conventional chemotherapeutics in vitro (A) Comparison of in vitro clonogenecity of the PLC/PRF/5 parental cells and sphere-forming cells. Cells were plated at a density of 2,000 cells/ well in 6-well plates and cultured for 1 week. At the end, cells were stained with crystal violet, photographed, and analyzed for their proliferation efficiency. Each experiment was performed in triplicate, and the representative examples were shown (Columns, mean; bars, SD; ${ }^{*}, P<0.01$ ). (B) The PLC/PRF/5 sphere-forming cells showed a drug resistance phenotype. The parental cells and sphere-forming cells were treated with cisplatin at the beginning of plating for $12 \mathrm{~h}$ or $24 \mathrm{~h}$ and 5 -Fu, gemcitabine, mitomycin and sorafenib for $36 \mathrm{~h}$. Cell survival was determined by MTT assay $(* *, P<0.01)$. 
Table 1 Tumorigenicity experiments of PLC/PRF/5 sphere-forming cells and parental cells in NOD/SCID mice

\begin{tabular}{|c|c|c|c|}
\hline Cell type & Cell numbers injected & Tumor incidence $^{\dagger}$ & ${\text { Latency }(\text { days })^{\ddagger}}^{*}$ \\
\hline \multirow[t]{9}{*}{ Sphere-forming cells of the 3rd generation } & $2 \times 10^{2}$ & $0 / 3$ & - \\
\hline & $5 \times 10^{2}$ & $1 / 3$ & 35 \\
\hline & $1 \times 10^{3}$ & $3 / 3$ & 23 \\
\hline & $2.5 \times 10^{3}$ & $3 / 3$ & 23 \\
\hline & $5 \times 10^{3}$ & $3 / 3$ & 20 \\
\hline & $1 \times 10^{4}$ & $3 / 3$ & 16 \\
\hline & $5 \times 10^{4}$ & $2 / 2$ & 9 \\
\hline & $1 \times 10^{5}$ & $2 / 2$ & 7 \\
\hline & $2 \times 10^{5}$ & $2 / 2$ & 7 \\
\hline \multirow[t]{3}{*}{ Sphere-forming cells of the 6th generation } & $2 \times 10^{2}$ & $0 / 3$ & - \\
\hline & $5 \times 10^{2}$ & $2 / 3$ & 49 \\
\hline & $1 \times 10^{3}$ & $3 / 3$ & 28 \\
\hline \multirow[t]{3}{*}{ Sphere-forming cells of the 9th generation } & $2 \times 10^{2}$ & $0 / 3$ & - \\
\hline & $5 \times 10^{2}$ & $1 / 3$ & 40 \\
\hline & $1 \times 10^{3}$ & $3 / 3$ & 30 \\
\hline \multirow[t]{5}{*}{ PLC/PRF/5 parental cells } & $5 \times 10^{4}$ & $0 / 3$ & - \\
\hline & $1 \times 10^{5}$ & $0 / 3$ & - \\
\hline & $2 \times 10^{5}$ & $2 / 3$ & 30 \\
\hline & $5 \times 10^{5}$ & $3 / 3$ & 16 \\
\hline & $1 \times 10^{6}$ & $2 / 2$ & 9 \\
\hline
\end{tabular}

${ }^{\dagger}$ The number of tumors detected/number of injections.

${ }^{\ddagger}$ Approximate number of days from tumor cell injection to appearance of a tumor.

5 sphere-forming cells are OV6 positive (Figure 4A). Then, liver CSCs related proteins, including Oct3/4, OV6, EpCAM, CD133 and CD44 were examined by Western blotting. The results displayed that all of them were significantly increased as compared to the parental cells (Figure 4B), denoting that the PLC/PRF/5 spheres possess stem cell-like properties. We also compared the expression of candidate CSC markers CD133 and CD44 between the parental and sphere-forming cells of HepG2 and MHCC $97 \mathrm{H}$ by immunofluorescent labelling. It was found that CD44 expression was obviously enriched in HepG2 and MHCC97H sphere-forming cells compared with their parental cells (Additional file 2, Figure S2).

To further explore the biological properties of liver CSCs, the Stem Cell RT ${ }^{2}$ ProfilerTM PCR array was performed. This array profiles the expression of 84 genes related to the identification, growth and differentiation of stem cells. Among the significantly distinguishing genes, we noted the DTX1 and Ep300, CSL-independent Notch signaling pathway related genes, were 4.24-fold and 2.36fold, respectively, more abundant in the spheres than those in the control. Consistent results were confirmed by Western blotting (Figure 5A). To determine the role of CSL-independent Notch signals, blocking Notch pathway was performed by a gamma-secretase inhibitor MRK003. The secondary dissociated PLC/PRF/5 sphereforming cells were treated with $10 \mu \mathrm{M}$ MRK003 or DMSO control for 7 days. The inactivation of Notch1 and down-regulations of downstream target genes DTX1 and Ep300 were confirmed by Western blotting (Figure $5 \mathrm{~B})$. The sphere formation ability of the MRK003-treated groups was significantly inhibited in comparison to the DMSO-treated controls (Figure $5 \mathrm{C}$ ). The results indicated that the CSL-independent Notch signaling pathway might play an important role in liver CSCs and MRK003 could partly eliminate the stem-like cells.

\section{Discussion}

There are two classical models of carcinogenesis [29,30]. One is the stochastic model, which is based on the belief that most of tumor cells are capable of extensive proliferation and contribute substantially to tumor maintenance; carcinogenesis could results from the random mutations and the subsequent clonal selections. The other is the hierarchical model, which is based on the belief that there are hierarchical differences among tumor cells, and only a small number of specific cells capable of extensive proliferation can contribute to carcinogenesis. The discovery of CSCs in solid tumors strongly supports the hierarchical model. The CSC hypothesis considers that CSCs subsets are located in the top of the hierarchical structure of tumor cells and directly affect the organization and construction of lower hierarchical cells. Therefore, the identification of tumorigenic liver CSCs could provide new insight into the HCC tumorigenic process and possibly bear great therapeutic implications. 


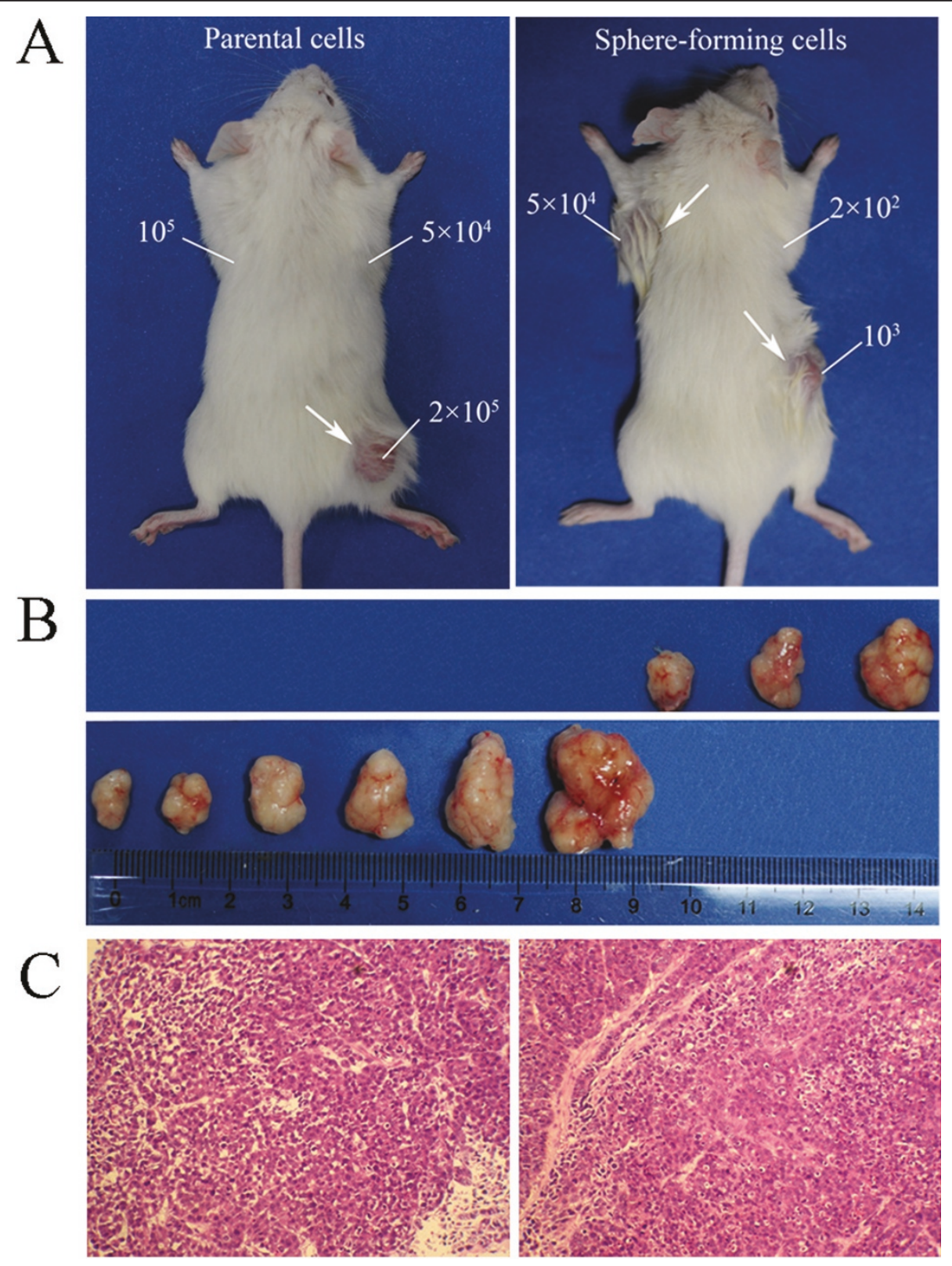

Figure 3 Sphere-forming cells exhibited high tumorigenicity in vivo. (A) The representative examples of xenograft tumors formed after subcutaneous injection with the PLC/PRF/5 parental cells and sphere-forming cells. (B) The top row shows the nodules formed by injecting $2 \times 10^{5}$, $5 \times 10^{5}$ and $1 \times 10^{6} \mathrm{PLC} / \mathrm{PRF} / 5$ parental cells, separately. The bottom row shows the nodules formed by injecting $5 \times 10^{2}, 1 \times 10^{3}, 2.5 \times 10^{3}, 5 \times 10^{3}$, $1 \times 10^{4}$ and $5 \times 10^{4} \mathrm{PLC} / \mathrm{PRF} / 5$ sphere-forming cells, separately. (C) H\&E staining revealed that the histological features of xenograft tumors induced by the PLC/PRF/5 sphere-forming cells were similar to those induced by the parental cells.

Usually, the isolation and identification of liver CSCs involve two types of methods: one is based on the sorting of side population (SP) cells that can exclude the hoechst 33342 dye [31]. However, Hoechst 33342 is cytotoxic; consequently, SP cells are protected by their membrane transport properties, whereas unprotected non-SP cells suffer toxicity and are unable to grow. Thus the differing tumor-initiation abilities of SP and non-SP cells are most likely due to an artifact of Hoechst 33342 toxicity, rather than due to intrinsic stem-cell properties [32]. The other type includes the fluorescence activated cell sorting (FACS) and the magnetic activated cell sorting (MACS), which are based on cell surface markers. The proposed markers for liver CSCs include CD133, CD90, CD44, CD13, EpCAM and OV6, on the basis of the hypothesis that CSCs are originated from somatic stem cells and 


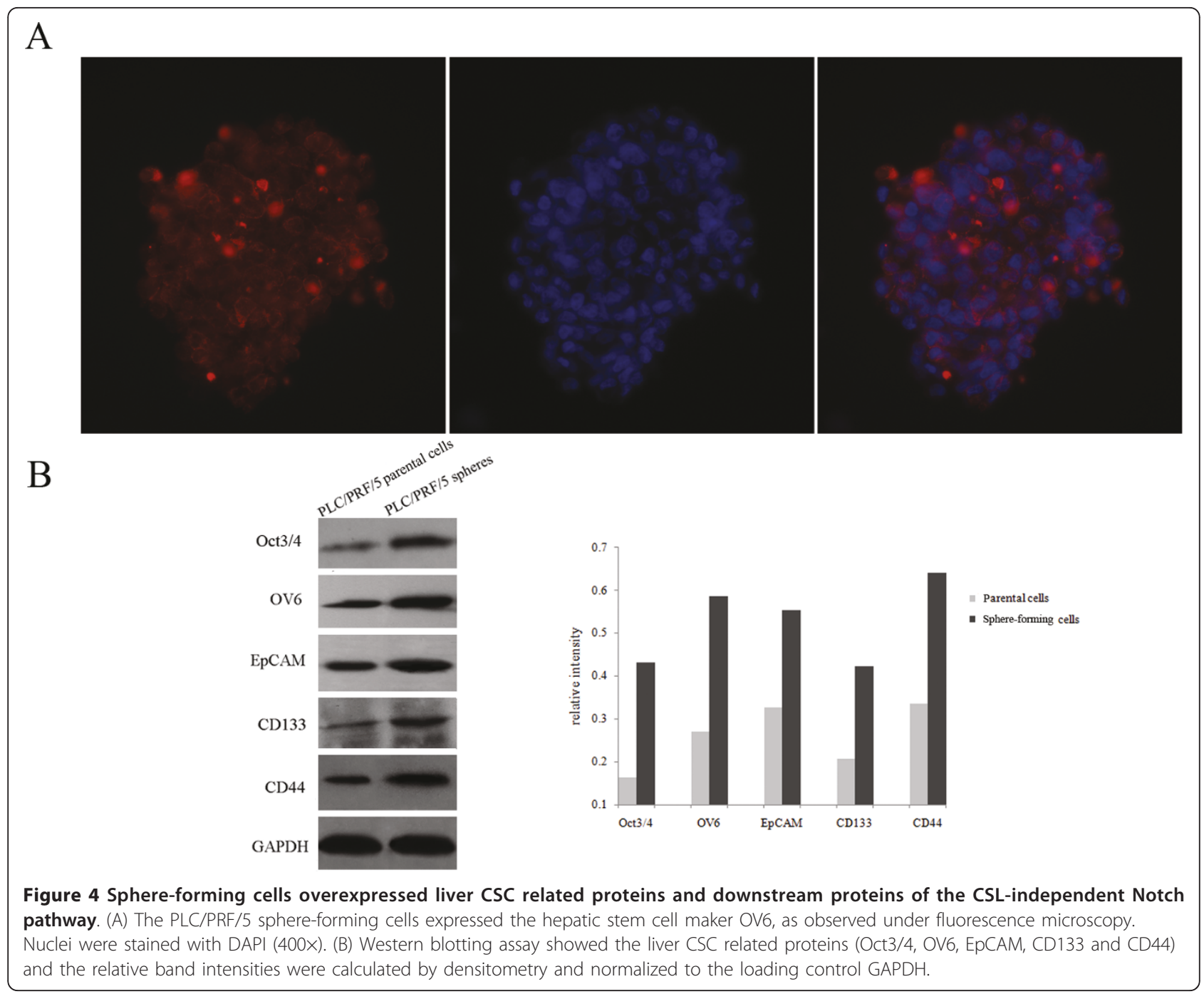

accordingly express the same surface markers [33,34]. Although they have been reported to be used to enrich CSC fraction, their sensitivity and specificity for identifying liver CSCs are being challenged. For example, Kimura et al reported $\mathrm{CD} 133^{+}$fraction in Hep3B and Huh7 were $16.8 \%$ and $2.7 \%$, respectively [7], whereas some other groups reported more than $90 \%$ in Hep3B and $60 \%$ in Huh7 $[5,6]$. In Huh7 the CD13-positive cells typically existed in a CD133 ${ }^{\text {strong }}$ fraction, but in PLC/PRF/5 the CD13-positive cells were CD133-negative [9]. Some researchers indicated that different culture conditions and differentiated degree of the cells, especially the latter, were important factors. The roles of these phenotypes in defining functionally distinct populations of cells from progenitor to differentiated hepatocytes need to be systemically studied.

Recently, sphere culture has been increasingly used as a method for enriching stem cells which relies on their property of anchorage independent growth. Researchers have reported the application of sphere culture to isolate, enrich, maintain or expand potential CSC subpopulations from various types of cancers [18-25]. The sphere-forming cells from primary tumors, such as breast cancer and ovarian cancer, showed stem-like properties and expressed their CSC markers $[19,23]$. It is generally agreed that, like all stem cells, the tumor sphere-forming cells are capable of proliferation, selfrenewal and possess higher tumorigenicity. Using neural crest stem cell conditions and sphere formation system, Hansford et al [35] for the first time successfully expanded tumor cells both from low-risk neuroblastomas and from the bone marrow metastases of high-risk tumors. The latter formed metastatic tumors in a murine xenograft model with as few as 10 cells and could also be serially passaged [35]. To our knowledge, there have been few reports on the isolation and long-term propagation of liver CSCs by the method of sphere culture. 


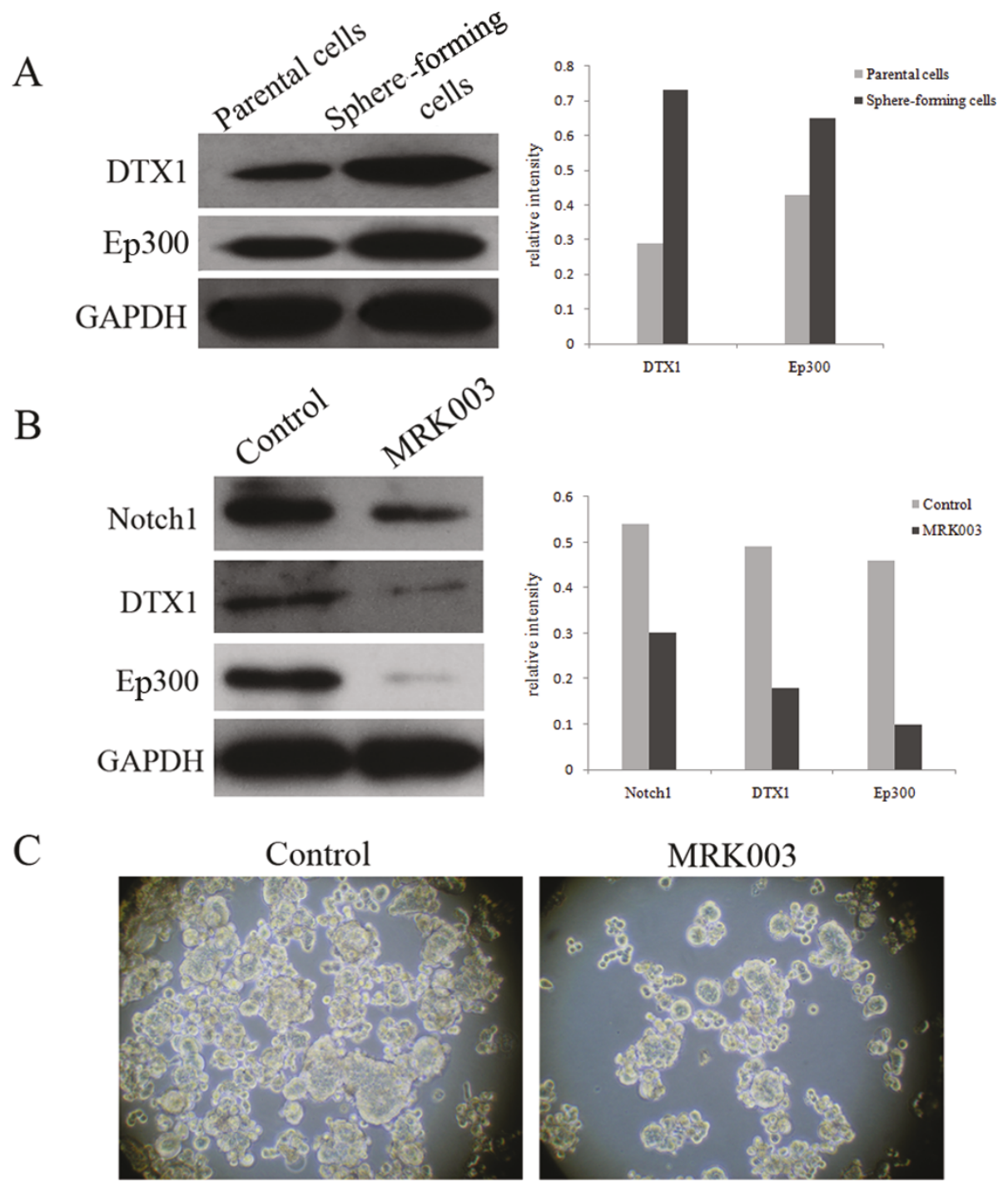

Figure 5 CSL-independent Notch signaling pathway might play a role in liver CSCs and MRK003 could partly eliminate the stem-like cells. (A) The downstream proteins levels of the CSL-independent Notch pathway (DTX1 and Ep300) were significantly increased in the PLC/ PRF/5 spheres, compared with the parental cell line. GAPDH was used as a loading control. (B) $10 \mu \mathrm{M}$ MRK003 could reduce the activation of Notch1 and downstream components of CSL-independent Notch signaling pathway DTX1 and Ep300 were consequently decreased. (C) The secondary dissociated PLC/PRF/5 sphere-forming cells were treated with $10 \mu \mathrm{M}$ MRKO03 or DMSO control for 7 days. The sphere formation ability of the MRKO03-treated groups were significantly inhibited in comparison to DMSO-treated controls.

In the present study, using stem cell conditioned culture system, we tested three human hepatoma cell lines, PLC/ PRF/5, MHCC97H and HepG2. Cells are plated at a low density ( $<5000$ cells/well in 6-well plate) to avoid spontaneous cell aggregation. The three cell lines could form clonal nonadherent 3-D spheres, and without exceptions, could also be serially passaged. We evaluated the PLC/ $\mathrm{PRF} / 5$ sphere-forming cells for their stemness characteristics. It has been showed that they were capable of selfrenewal, proliferation, drug resistance, and overexpressing liver CSC related proteins. Xenotransplantation is the gold standard for evaluating tumorigenicity of tumor cells. We tested the third, sixth and ninth generations of the PLC/ $\mathrm{PRF} / 5$ sphere-forming cells for their tumor initiating capability. It was demonstrated that as few as 500 cells from the PLC/PRF/5 spheres were able to form a tumor when subcutaneously injected into NOD/SCID mice, while $2 \times$ $10^{5}$ parental cells were needed. This was 400 times higher than that of sphere-forming cells. Moreover, the tumor initiating capability was not decreased as the spheres were passaged. Similar CSC properties of self-renewal, strongly proliferation, drug resistance and tumorigenicity are also found in the MHCC $97 \mathrm{H}$ and HepG2 spheres. Indeed, the HepG2 parental cells at $10^{6}$ cells/mouse could not form 
visible xenografts in nude mice, but its sphere-forming cells at the same amount of cells could form xenograft tumors, suggesting the tumorigenic efficacies of sphereforming cells were enhanced compared with the parental cancer cells.

To further explore the CSC properties of sphere-forming cells, we examined the sensitivity of sphere-forming cells to chemotherapeutics and the expression of candidate CSC markers. The PLC/PRF/5 sphere-forming cells exhibited general resistance to cisplatin, 5-Fu, gemcitabine, mitomycin and sorafenib, and showed higher survival percentages compared with its parental cells. Synchronously, we found that CD44 expression was obviously enriched in HepG2 and MHCC97H sphere-forming cells compared with their parental cells. CD44 is a polymorphic family of immunologically related cell surface proteoglycans and glycoproteins, normally takes part in cell-cell and cellmatrix adhesion interactions, which is involved in cancer cell migration, proliferation and metastasis. Accordingly, CD44 expression enrichment in sphere-forming cells may account for their increased survival ability and tumorigenicity. Therefore, we propose that the nonadherent tumor spheres cultured in serum-free condition possess liver CSC properties. This long-term culture system may also provide the means of further purifying and functionally characterizing the biological properties of the liver CSC fraction, with the goal of developing new therapeutic strategies directed specifically against liver CSCs.

Accumulating evidence has been established that the Notch signaling pathway plays vital and universal roles not only in cell differentiation, embryonic development and tissue self-renewal, but also in pathogenesis of some types of human cancers and genetic disorders. Recent advancements have further revealed that the Notch signals produce a marked effect either in stem cells or CSCs. The activated Notch signals can inhibit hematopoietic stem cell differentiation and maintain their pluripotency [36,37], and maintaining the stem cell population in several solid tissue types, including several neuroectodermal tissues [38]. Only when the Notch signals are activated the cancer stem cell activity could be enhanced to promote intestinal tumor formation [39]. Generally, the Notch signaling pathway is mediated in two different pathways. One is through CSL-DNA binding proteins; the other is the CSL-independent pathway. DTX1 (Deltex-1) is an important transcriptional regulator that is downstream of the Notch receptor in the CSL-independent Notch signaling pathway [40]. Ep300, also known as p300, is a transcriptional co-activator protein. It functions as a histone acetyltransferase that regulates transcription via chromatin remodeling and is important in the processes of cell proliferation and differentiation. It has been reported that Ep300 can work as a transcriptional co-activator of DTX1. Yamamoto et al [41] reported that DTX1 inhibited the transcriptional activation of the neural-specific helix-loop-helix type transcription factor MASH1 by binding to Ep300. This mechanism is likely responsible for the differentiation inhibition of neural progenitor cells. In our study, the result of stem cell microarrays showed that DTX1 and Ep300 were highly expressed in liver cancer stem-like cells. This was further confirmed by Western blotting. Although the molecular mechanism and function of the CSL-independent Notch signaling pathway have not been elucidated, and little has been known about its involvement in HCC, we suppose that the CSL-independent Notch signaling pathway play an important role in the differentiation and propagation of liver CSCs.

\section{Conclusions}

In summary, the study demonstrated that the nonadherent tumor spheres from human hepatoma cell lines which are cultured in stem cell conditioned medium possess liver cancer stem cell properties, and the CSLindependent Notch signaling pathway may play a role in the differentiation and propagation of liver CSCs.

\section{Additional material}

Additional file 1: Figure S1. Tumorigenic efficacies of the hepatoma sphere-forming cells. The hepatoma parental cells and sphere-forming cells were injected into nude mice subcutaneously at the indicated cell concentrations. At day $30^{\text {th }}$ after injection, mice were sacrificed and tumors were removed and compared in size.

Additional file 2: Figure S2. Expression of candidate CSC markers in hepatoma sphere-forming cells. Confocal immunofluorescent staining showed that CD44 expression was enriched obviously in HepG2 and $\mathrm{MHCC} 97 \mathrm{H}$ sphere-forming cells compared with their parental cells. Nuclei were stained with DAPI (400x).

\section{Acknowledgements}

This study was supported by National High-Tech Research and Development Program of China (No. 2007AA02Z461), China National Key Projects for Infectious Disease (2008ZX10002-021) and National Natural Science Foundation of China (No. 30672002, 30801342).

\section{Author details}

${ }^{1}$ Department of Molecular Oncology, Eastern Hepatobiliary Surgery Hospital, Second Military Medical University, Shanghai, China. ${ }^{2}$ Department of HepatoBiliary-Pancreato-Vascular Surgery, the First affiliated Hospital of Xiamen University, Xiamen, China.

\section{Authors' contributions}

Lu C conceived the study and participated in the study design, performance, coordination and manuscript writing. YMZ participated in the study performance and writing. MCW participated in the study design and coordination. ZFY participated in the study design, coordination and writing. $B B Z$, Jian $L, W X, R X Z$, Jing $L, Y Z$, Lei $C$ and $H H Q$ performed the research. All authors have read and approved the final manuscript.

\section{Competing interests}

The authors declare that they have no competing interersts.

Received: 21 October 2010 Accepted: 14 June 2011

Published: 14 June 2011 


\section{References}

1. Parkin DM: Global cancer statistics in the year 2000. Lancet Oncol 2001, 2:533-543

2. El-Serag HB, Rudolph KL: Hepatocellular carcinoma: epidemiology and molecular carcinogenesis. Gastroenterology 2007, 132:2557-2576.

3. Altekruse SF, McGlynn KA, Reichman ME: Hepatocellular carcinoma incidence, mortality, and survival trends in the United States from 1975 to 2005. J Clin Oncol 2009, 27:1485-1491.

4. Yang ZF, Ho DW, Ng MN, Lau CK, Yu WC, Ngai P, Chu PW, Lam CT, Poon RT, Fan ST: Significance of CD90+ cancer stem cells in human liver cancer. Cancer Cell 2008, 13:153-166.

5. Ma S, Chan KW, Hu L, Lee TK, Wo JY, Ng IO, Zheng BJ, Guan XY: Identification and characterization of tumorigenic liver cancer stem/ progenitor cells. Gastroenterology 2007, 132:2542-2556

6. Zhu Z, Hao X, Yan M, Yao M, Ge C, Gu J, Li J: Cancer stem/progenitor cells are highly enriched in CD133+CD44+ population in hepatocellular carcinoma. Int J Cancer 2010, 126:2067-2078.

7. Kimura O, Takahashi $T$, Ishii N, Inoue $Y$, Ueno $Y$, Kogure T, Fukushima $K$, Shiina M, Yamagiwa Y, Kondo Y, Inoue J, Kakazu E, Iwasaki T, Kawagishi N, Shimosegawa T, Sugamura K: Characterization of the epithelial cell adhesion molecule (EpCAM)+ cell population in hepatocellular carcinoma cell lines. Cancer Sci 2010, 101:2145-2155.

8. Yang W, Yan HX, Chen L, Liu Q, He YQ, Yu LX, Zhang SH, Huang DD, Tang L, Kong XN, Chen C, Liu SQ, Wu MC, Wang HY: Wnt/beta-catenin signaling contributes to activation of normal and tumorigenic liver progenitor cells. Cancer Res 2008, 68:4287-4295.

9. Haraguchi $N$, Ishii $H$, Mimori K, Tanaka F, Ohkuma M, Kim HM, Akita H akiuchi D, Hatano H, Nagano H, Barnard GF, Doki Y, Mori M: CD13 is a therapeutic target in human liver cancer stem cells. J Clin Invest 2010, 120:3326-3339.

10. Sell S, Leffert HL: Liver cancer stem cells. J Clin Oncol 2008, 26:2800-2805

11. Wu Y, Wu PY: CD133 as a marker for cancer stem cells: progresses and concerns. Stem Cells Dev 2009, 18:1127-1134.

12. Salnikov AV, Kusumawidjaja G, Rausch V, Bruns H, Gross W, Khamidjanov A, Ryschich E, Gebhard MM, Moldenhauer G, Büchler MW, Schemmer P, Herr I: Cancer stem cell marker expression in hepatocellular carcinoma and liver metastases is not sufficient as single prognostic parameter. Cancer Lett 2009, 275:185-193.

13. Reynolds BA, Weiss S: Clonal and population analyses demonstrate that an EGF-responsive mammalian embryonic CNS precursor is a stem cell. Dev Biol 1996, 175:1-13.

14. Dontu G, Abdallah WM, Foley JM, Jackson KW, Clarke MF, Kawamura MJ, Wicha M: In vitro propagation and transcriptional profiling of human mammary stem/progenitor cells. Genes Dev 2003, 17:1253-1270.

15. Suzuki A, Oyama K, Fukao K, Nakauchi H, Taniguchi H: Establishment of clonal colony-forming assay system for pancreatic stem/progenitor cells. Cell Transplant 2002, 11:451-453.

16. Bez A, Corsini E, Curti D, Biggiogera M, Colombo A, Nicosia RF, Pagano SF Parati EA: Neurosphere and neurosphere-forming cells: morphological and ultrastructural characterization. Brain Res 2003, 993:18-29.

17. Shi X, Gipp J, Bushman W: Anchorage-independent culture maintains prostate stem cells. Dev Biol 2007, 312:396-406.

18. Singh SK, Clarke ID, Terasaki M, Bonn VE, Hawkins C, Squire J, Dirks P: Identification of a cancer stem cell in human brain tumors. Cancer Res 2003, 63:5821-5828.

19. Ponti D, Costa A, Zaffaroni N, Pratesi G, Petrangolini G, Coradini D, Pilotti S, Pierotti MA, Daidone M: Isolation and in vitro propagation of tumorigenic breast cancer cells with stem/progenitor cell properties. Cancer Res 2005, 65:5506-5511.

20. Gibbs CP, Kukekov VG, Reith JD, Tchigrinova O, Suslov ON, Scott EW, Ghivizzani SC, Ignatova TN, Steindler DA: Stem-like cells in bone sarcomas: implications for tumorigenesis. Neoplasia 2005, 7:967-976.

21. Fang D, Nguyen TK, Leishear K, Finko R, Kulp AN, Hotz S, Van Belle PA Xu X, Elder DE, Herlyn M: A tumorigenic subpopulation with stem cell properties in melanomas. Cancer Res 2005, 65:9328-9337.

22. Gou S, Liu T, Wang C, Yin T, Li K, Yang M, Zhou J: Establishment of clonal colony-forming assay for propagation of pancreatic cancer cells with stem cell properties. Pancreas 2007, 34:429-435.

23. Zhang S, Balch C, Chan MW, Lai HC, Matei D, Schilder JM, Yan PS, Huang TH, Nephew KP: Identification and Characterization of Ovarian
Cancer-Initiating Cells from Primary Human Tumors. Cancer Res 2008, 68:4311-4320.

24. Fujii H, Honoki K, Tsujiuchi T, Kido A, Yoshitani K, Takakura Y: Sphereforming stem-like cell populations with drug resistance in human sarcoma cell lines. Int J Oncol 2009, 34:1381-1386.

25. Rappa G, Mercapide J, Anzanello F, Prasmickaite L, Xi Y, Ju J, Fodstad O, Lorico A: Growth of cancer cell lines under stem cell-like conditions has the potential to unveil therapeutic targets. Exp Cell Res 2008, 314:2110-2122.

26. Dunsford HA, Sell S: Production of monoclonal antibodies to preneoplastic liver cell populations induced by chemical carcinogens in rats and to transplantable Morris hepatomas. Cancer Res 1989, 49:4887-4893.

27. Strain AJ, Crosby HA, Nijjar S, Kelly DA, Hubscher SG: Human liver-derived stem cells. Semin Liver Dis 2003, 23:373-384.

28. Van Den Heuvel MC, Slooff MJ, Visser L, Muller M, De Jong KP, Poppema S, Gouw AS: Expression of anti-OV6 antibody and anti-N-CAM antibody along the biliary line of normal and diseased human livers. Hepatology 2001, 33:1387-1393.

29. Michelson S, Slate D: Emergence of the drug-resistant phenotype in tumor subpopulations: a hybrid model. J Natl Cancer Inst 1989, 81:1392-1401.

30. Perryman SV, Sylvester KG: Repair and regeneration: opportunities for carcinogenesis from tissue stem cells. J Cell Mol Med 2006, 10:292-308.

31. Chiba T, Kita K, Zheng YW, Yokosuka O, Saisho H, Iwama A, Nakauchi H, Taniguchi $\mathrm{H}$ : Side population purified from hepatocellular carcinoma cells harbors cancer stem cell-like properties. Hepatology 2006 , 44:240-251.

32. Shen $G$, Shen F, Shi Z, Liu W, Hu W, Zheng X, Wen L, Yang X: Identification of cancer stem-like cells in the C 6 glioma cell line and the limitation of current identification methods. Vitro Cell Dev Biol Anim 2008, 44:280-289.

33. Pardal R, Clarke MF, Morrison SJ: Applying the principles of stem-cell biology to cancer. Nat Rev Cancer 2003, 3:895-902

34. Marx J: Cancer research. Mutant stem cells may seed cancer. Science 2003, 301:1308-1310

35. Hansford LM, McKee AE, Zhang L, George RE, Gerstle JT, Thorner PS, Smith KM, Look AT, Yeger H, Miller FD, Irwin MS, Thiele CJ, Kaplan DR: Neuroblastoma cells isolated from bone marrow metastases contain a naturally enriched tumor-initiating cell. Cancer Res 2007, 67:11234-11243.

36. Duncan AW, Rattis FM, DiMascio LN, Congdon KL, Pazianos G, Zhao C, Yoon K, Cook JM, Willert K, Gaiano N, Reya T: Integration of Notch and Wnt signaling in hematopoietic stem cell maintenance. Nat Immunol 2005, 6:314-322

37. Stier S, Cheng T, Dombkowski D, Carlesso N, Scadden DT: Notch1 activation increases hematopoietic stem cell self-renewal in vivo and favors lymphoid over myeloid lineage outcome. Blood 2002, 99:2369-2378.

38. Pinnix CC, Herlyn M: The many faces of Notch signaling in skin-derived cells. Pigment Cell Res 2007, 20:458-465.

39. van Es JH, van Gijn ME, Riccio O, van den Born M, Vooijs M, Begthel $H$, Cozijnsen M, Robine S, Winton DJ, Radtke F, Clevers H: Notch/gammasecretase inhibition turns proliferative cells in intestinal crypts and adenomas into goblet cells. Nature 2005, 435:959-963.

40. Martinez Arias A, Zecchini V, Brennan K: CSL-independent Notch signaling: a checkpoint in cell fate decisions during development? Curr Opin Genet Dev 2002, 12:524-533.

41. Yamamoto N, Yamamoto S, Inagaki F: Role of Deltex-1 as a transcriptional regulator downstream of the Notch receptor. J Biol Chem 2001, 276:45031-45040.

\section{Pre-publication history}

The pre-publication history for this paper can be accessed here: http://www.biomedcentral.com/1471-230X/11/71/prepub

doi:10.1186/1471-230X-11-71

Cite this article as: Cao et al:: Sphere-forming cell subpopulations with cancer stem cell properties in human hepatoma cell lines. BMC Gastroenterology 2011 11:71. 